\title{
La Crioterapia con nitrógeno líquido fue más efectiva que el acido salicílico en el tratamiento de verrugas cutáneas
}

Cryotherapy with liquid nitrogen was more effective than salicylic acid in the treatment of cutaneous warts

Bruggink SC y col. CMAJ 2010;182(15):1624-30

\section{Objetivos}

Comparar la efectividad del tratamiento de las verrugas cutáneas mediante crioterapia con nitrógeno líquido contra la aplicación tópica de acido salicílico.

\section{Diseño, lugar y pacientes}

Ensayo clínico aleatorizado, simple ciego (investigadores). Se realizo en Holanda. El reclutamiento de pacientes se inicio en mayo de 2006 y el seguimiento fue de 13 meses. Participaron 30 centros de atención primaria que a su vez identificaron 250 pacientes (de entre 4 y 79 años) con verrugas cutáneas de cualquier localización que no hubiesen recibido atención profesional en el último año. Se excluyeron pacientes inmunocomprometidos y verrugas genitales, seborreicas o mayores a un centímetro de diámetro. Las lesiones fueron estratificadas* de acuerdo a localización (planta de pié u otra localización) y número de lesiones (mas o menos de seis lesiones). La aleatorización se generó por computadora y el enmascaramiento de la asignación, por sobres opacos*. El análisis se efectuó por intención de tratar modificado*.

\section{Intervención}

Se establecieron tres ramas de tratamiento: los pacientes aleatorizados a crioterapia ( $\mathrm{B} 80$ ), recibían topicaciones con nitrógeno líquido cada dos semanas; los pacientes aleatorizados a ácido salicílico (gel al 40\%; n 84) se autoaplicaban diariamente el producto; por último el grupo control ( $\mathrm{n} 86$ ) mantenía conducta expectante (n 86). En todos los casos se suspendía el tratamiento al desaparecer las lesiones. Al completarse la semana 13, los pacientes podían optar por cambiar de tratamiento en caso de lesiones persistentes.

\section{Medición de resultados principales}

El resultado primario fue el porcentaje de pacientes cuyas lesiones resultaron totalmente curadas a la semana $13^{\circ}$. Los resultados secundarios evaluaron efectos adversos, satisfacción y carga del tratamiento.

\section{Resultados principales}

Los resultados más relevantes se detallan en la tabla 1. Los resultados no se modificaron al analizar los pacientes perdidos en el seguimiento como no curados. En líneas generales, los pacientes del grupo crioterapia experimentaron mayores efectos adversos (dolor, ampollas, cicatrices, irritación y pigmentación cutánea), sin embargo reportaron una menor sobrecarga y mayor satisfacción relacionada con el tratamiento.

Tabla 1: Tasas de curación (pacientes sin lesiones) a las 13 semanas

\begin{tabular}{|c|c|c|c|c|c|}
\hline \multirow[b]{2}{*}{ Variable } & \multicolumn{3}{|c|}{ Valores absolutos para cada grupo } & \multicolumn{2}{|c|}{$\begin{array}{l}\text { Valores relativos al comparar } \\
\text { crioterapla y ácido salicilico }\end{array}$} \\
\hline & $\begin{array}{l}\text { Crioterapia } \\
\% \text { (IC } 95 \% \text { ) }\end{array}$ & $\begin{array}{l}\text { Ac. Salicilico } \\
\%(\text { (IC } 95 \%)\end{array}$ & $\begin{array}{c}\text { Conducta expectante } \\
\% \text { (IC } 95 \%)\end{array}$ & $\operatorname{RR}($ (IC 95\%) & (IC 95\%) \\
\hline Total de pacientes (n 240) & $39(29$ a 51$)$ & 24 (16 a 35) & $16(9,5$ a 25$)$ & 1,62 (1,01 a 2,59) & $6,6(3,4$ a 145 \\
\hline Verrugas comunes (n 116) & 49 (34 a 64) & 15 (7 a 30) & 8 (2 a 25) & $3,17(1,42$ a 7,07$)$ & $3,0(1,9$ a 7,1$)$ \\
\hline Verrugas plantares ( $\mathrm{n}$ 124) & $30(17$ a 46$)$ & $33(20$ a 47$)$ & 23 (13 а 37) & $0,91(0,47$ a 1,76$)$ & - \\
\hline
\end{tabular}

\section{Conclusiones}

El tratamiento con crioterapia resultó más efectivo para verrugas comunes. Para verrugas plantares, no se encontraron diferencias clínicamente relevantes entre crioterapia, acido salicílico o conducta expectante luego de 13 semanas.

Fuente de financiamiento: Fundación Nacional Suiza y Fundación Robert Bosch.

\section{Comentario}

Las verrugas cutáneas son un problema muy frecuente de consulta en atención primaria, si bien tiene un curso de evolución benigno y con tendencia a autolimitarse ${ }^{1}$, generan disconfort en gran parte de los pacientes, la mayoría estéticos ${ }^{2}$. El tratamiento usual en nuestro medio consiste en la aplicación tópica de ácido salicílico ${ }^{3}$.

Este estudio, metodológicamente aceptable, nos muestra una eficacia superior para el tratamiento de las verrugas comunes con crioterapia, con el cual los pacientes estuvieron más satisfechos a pesar de los mayores efectos adversos. Se logro también observar diferente comportamiento de las verrugas con tasas de curación mayores en niños y en aquellas verrugas de reciente aparición $(<6$ meses).

Un punto importante a considerar al extrapolar estos resultados a nuestro medio es el hecho de la disponibilidad del tratamiento de crioterapia (que requiere disponibilidad del producto y consulta quincenal con un médico entrenado en la técnica de aplicación). Por otro lado no se han considerado los costos de las estrategias de intervención activa.

\section{Conclusiones de comentador}

Cuando se encuentre accesible, debemos tener presente a la crioterapia como método más eficiente en el tratamiento de las verrugas comunes localizadas en las manos y otras parte del cuerpo. Por el momento no existe evidencia de superioridad de ninguno de los tratamientos sobre las verrugas plantares.

Nadia Musarella y Gabriel Villalón [ Servicio de Medicina Familiar y Comunitaria del Hospital Italiano de Buenos Aires. nadia.musarella@ hospitalitaliano.org.ar ]

Musarella N. La Crioterapia con nitrógeno líquido fue más efectiva que el acido salicílico en el tratamiento de verrugas cutáneas. Evid Act Pract Ambul. Oct-Dic 2011;14(4):132. Comentado de: Bruggink SC, Gussekloo J, Berger MY y col. Cryotherapy with liquid nitrogen versus topical salicylic acid application for cutaneous warts in primary care: randomized controlled trial. CMAJ 2010;182(15):1624-30. PMID 20837684.

\section{Referencias}

1. Rubinstein E, Di Paolo R. Ectoparasitosis, micosis superficiales y otras infecciones cutáneas. Capítulo 245. Sección 22 Problemas dermatológicos frecuentes. En: "Medicina Familiar y Practica Ambulatoria". Editores Rubinstein A y Terrasa S. Editorial Médica Panamericana, Enero de 2006. pag 1853.

2. Gibbs S, Harvey I. Topical treatments for cutaneous warts. Cochrane Database of Systematic Reviews 2006, Issue 3. Art. No.: CD001781. DOI: 10.1002/14651858.CD001781. pub2 3. Fernández-Obanza Windscheid E, García Sieiro R. Verrugas cutáneas. Guías Clínicas en Atención Primaria. Guías Clínicas 2004;4(21). 